\title{
Principal Component Analysis (PCA) in the Context of Radar Polarimetry
}

\author{
W.-M. Boerner ${ }^{1}$, E. Lüneburg ${ }^{2}$, and A. Danklmayer ${ }^{3}$ \\ ${ }^{1}$ University of Illinois at Chicago, USA \\ ${ }^{2}$ EML Consultants, Germany \\ ${ }^{3}$ German Aerospace Center, Germany
}

\begin{abstract}
Statistical and computational techniques for revealing the internal structure that underlies the set of random correlated data exists in a great variety at present; and target decomposition theorems, either in the coherent or incoherent formulation, are well established. In spite of this fact a rather innovative and new concept is presented in this contribution. In turn the Principal Component Analysis (PCA) is considered to possibly add value to existing approaches, and it allows for an interpretation of polarimetric synthetic aperture radar measurements using variables obtained via linear transformation. Starting with the Sinclair backscatter matrix S which will be further transformed into the so called target feature vector by stacking column elements of $\mathrm{S}$ and generating the covariance matrices averaged over a certain pixel array, we show, how the Sinclair backscatter matrix is decomposed into the sum of a maximum of four $2 \times 2$ elementary point scatter matrices which are weighted by the principal components, whereas the variances of these components agree with the eigenvalues of the covariance matrix. This mathematical development defines a decomposition which expresses scattering mechanism from distributed targets in terms of scattering matrices via an incoherent step.
\end{abstract}

\section{INTRODUCTION}

In radar and optical polarimetry there exists essentially two different methods to characterize polarimetric scattering properties of plane electromagnetic waves scattered by randomly distributed targets using second order multivariate statistics [1-3]. The first is the Kennaugh matrix which is used for finding solutions for maximal and minimal power transfer between transmitting and receiving antennas. The second is the covariance matrix of which the analysis is used for entropy and variance considerations and for the generation of uncorrelated random variables.

The basic problem of principal component analysis $[4,5,8]$ is how to find a suitable representation of multivariate data in order to make the essential structure more visible and to identify any distinct feature. The main purpose of PCA is to convert a set of possibly correlated random variables into new uncorrelated variables.

In radar polarimetry and interferometry, the principal component analysis is known as target decomposition theory, and relevant contributions are due to [1,2,6,9-13] and many others. Only covariance matrices rather than correlation matrices are used. Considerable emphasis is paid to the interpretation of the coefficients and the eigenvectors of the covariance matrix. Confusion still prevails with respect to the correct interpretation of the principal components (the new uncorrelated orthonormal random variables) and the only superficially related concepts of the classical Sinclair/Kennaugh and the Jones/Mueller matrices. The principal component analysis (PCA) relies on two basic requirements of a random vector: (i) to find linear functions of the components of the random vector having maximum variance in subsequent steps; and (ii) that these new random variables should be orthonormal and uncorrelated. These requirements imply that the first few components carry most of the total variation of the original random vector and are representative for multivariate random processes.

\section{PRINCIPLE COMPONENT ANALYSIS (PCA)}

With principal component analysis one tries to find new variables which are uncorrelated but not necessary independent. As a starting point the scattering matrix represented by the $2 \times 2 \mathrm{~S}$-matrix is considered, describing completely the polarization transforming properties of a target at a single frequency in the reference direction.

$$
S(t)=\left(\begin{array}{ll}
S_{H H}(t) & S_{H V}(t) \\
S_{V H}(t) & S_{V V}(t)
\end{array}\right)
$$


The indices of the matrix elements represent the transmit and receive polarization of the plane electromagnetic wave and $t$ stands for time or ensemble values. The vec operator [7] is used to arrive at the target feature vector $k$ given by

$$
k_{4}(t)=\operatorname{vec} S(t)=\left[\begin{array}{l}
S_{H H}(t) \\
S_{V H}(t) \\
S_{H V}(t) \\
S_{V V}(t)
\end{array}\right]
$$

where the "vec" operator can be considered as a simple stacking of the columns of the scattering matrix $S(t)$. This target feature vector is used to calculate the $4 \times 4$ covariance matrix explicitly according to

$$
C_{4}=<k_{4}(t) k_{4}^{\dagger}(t)>.
$$

where the dagger symbol represents complex conjugation and transposition (Hermition conjugate) and the angle brackets denote the averaging of the outer product of $k_{4}(t)$.

Being Hermitian positive semidefinite, the covariance matrix $C_{4}$ can be unitarily diagonalized by a unitary matrix with non-negative eigenvalues.

$$
U^{-1} C_{4} U=\Lambda \equiv \operatorname{diag}\left[\lambda_{1}, \lambda_{2}, \lambda_{3}, \lambda_{4}\right] \text { with } U=\left[\hat{x}_{1}, \hat{x}_{2}, \hat{x}_{3}, \hat{x}_{4}\right] .
$$

$\lambda_{i}(i=1, \ldots, 4)$ are denoting the eigenvalues of the covariance matrix, and $x_{i}(i=1, \ldots, 4)$ are the eigenvectors, respectively. Now we introduce the new target feature vector $Z(t)$ by a linear transformation

$$
Z(t)=\left[\begin{array}{c}
z_{1} \\
z_{2} \\
z_{3} \\
z_{4}
\end{array}\right]=U^{\dagger} k(t)=\left[\begin{array}{c}
x_{1}^{\dagger} k(t) \\
x_{2}^{\dagger} k(t) \\
x_{3}^{\dagger} k(t) \\
x_{4}^{\dagger} k(t)
\end{array}\right] \text { or } k(t)=U \cdot Z(t)
$$

The components $z_{i}(i=1, \ldots, 4)$ are called the principal components (the new uncorrelated orthonormal random variables). They are uncorrelated new random variables and their variances are equal to the corresponding eigenvalues of $C_{4}$. Furthermore the spectral decomposition of the Covariance matrix is given by

$$
C_{4}=U \Lambda U^{\dagger}=\sum_{i=1}^{4} \lambda_{i} x_{i} x_{i}^{\dagger}=\sum_{i=1}^{4} \lambda_{i} C_{4, i}
$$

where $C_{4 i}$ are $4 \times 4$ covariance matrices of point targets with rank 1 . The reverse vec operator may be applied to $\boldsymbol{x}_{i}$ and the results can be interpreted as $2 \times 2$ elementary deterministic point targets $S_{i}$ with $\operatorname{span}\left(S_{i}\right)=1$.

$$
x_{i}=\operatorname{vec} S_{i}=\left[\begin{array}{l}
x_{i, 1} \\
x_{i, 2} \\
x_{i, 3} \\
x_{i, 4}
\end{array}\right] \Leftrightarrow S_{i}=\left[\begin{array}{ll}
x_{i, 1} & x_{i, 3} \\
x_{i, 2} & x_{i, 4}
\end{array}\right](i=1, \ldots, 4)
$$

and hence using the relation $k(t)=U Z(t)$

$$
S(t)=\left[\begin{array}{ll}
S_{x x} & S_{x y} \\
S_{y x} & S_{y y}
\end{array}\right]=\sum_{i=1}^{4} z_{i}(t)\left[\begin{array}{ll}
x_{i 1} & x_{i 3} \\
x_{i 2} & x_{i 4}
\end{array}\right]=\sum_{i=1}^{4} z_{i}(t) S_{i}
$$

This is an expansion of the scattering matrix into four uncorrelated point targets formed by the loading vectors or loading matrices with random coefficients $z_{i}(t)$. For reciprocal targets, the matrix $S(t)$ is symmetric; then the elementary point target matrices $S_{i}(t)$ are also symmetric, i.e., $x_{i 2}=x_{i 3}$ for all $i$.

The basic targets are orthonormal in the sense that

$$
\left(\operatorname{vec} S_{i}\right)^{\dagger} \operatorname{vec} S_{j}=x_{i}^{\dagger} x_{j}=\sum_{k=1}^{4} x_{i k}^{*} x_{j} k=\sum_{k=1}^{4}\left|x_{i k}\right|^{2} \delta_{i j}=\delta_{i j}
$$

for all $i, j=1, \ldots, 4$. 


\section{APPLICATION OF PCA TO POLARIMETRIC SAR DATA}

Calculations have been carried out following the mathematical developments presented in the previous chapter. A polarimetric SAR data set (L-band) was used and Fig. 1 shows a RGB-image of the testsite Oberpfaffenhofen, Germany. The different channels of the image are red for HH-, green for $\mathrm{HV}$-, and blue color for VV-polarization. The window size for the applied averaging of the covariance matrix was chosen $5 \times 5$ pixels. At the end of the calculations the result is the extension of the scattering matrix into the sum of four matrices (cf. right hand side of (8)). These matrices represent the product of the principal components $z_{i}(t)$ with the elementary point scatter matrix and the elements of these matrices were used for visualization of the results.

\section{RESULTS}

The image which is presented in Fig. 2 refers to the results that were obtained by the ICA. It was chosen to concentrate on the results of the principal components with the highest variance. One matrix of the four possible matrices has been used in order to generate the images.

In Fig. 2, the RGB image $\left(R=z_{4} x_{41}, G=z_{4} x_{44}, B=z_{4} x_{42}=z_{4} x_{43}\right)$ for the elements of the matrices obtained from the principal component with the highest variance multiplied with the corresponding point scatter matrix is presented. It has been observed that the RGB image in Fig. 2 has differences in comparison with the original data in Fig. 1. Grassland for instance appears brighter in the RGB image of Fig. 2 and has a better contrast to neighbouring regions. For a comparison see the lower right of Figs. 1 and 2, respectively.

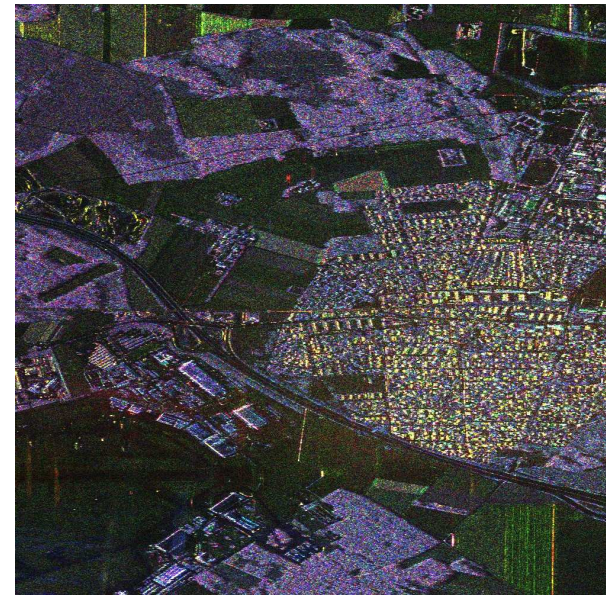

Figure 1: RGB image of the original data.

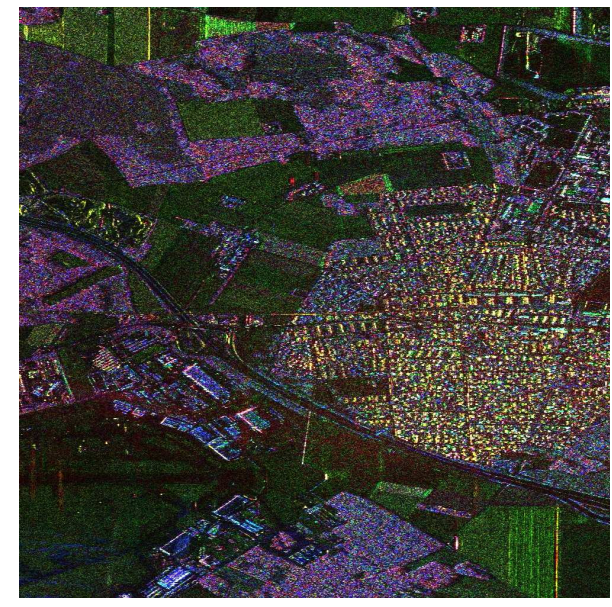

Figure 2: RGB image $\left(R=z_{4} x_{41}, G=z_{4} x_{44}\right.$, $\left.B=z_{4} x_{42}=z_{4} x_{43}\right)$.

\section{CONCLUSIONS}

Having a series of coherent observations given by the scattering matrix $S(t)$ the principal component analysis provides a link to the incoherent method of interpretation (covariance matrix analysis) and furthermore an equivalent coherent representation containing a maximum of 4 possible uncorrelated features. The target description can be formed using $z_{i}$ values and $S_{i}$ point scatter matrices. The proposed technique is one of a variety of statistical and computational techniques to reveal the internal structure that underlies the set of random correlated data. Further work has to be devoted to elaborate a quantitative analysis and comparison to existing methods. Moreover a statistical analysis of the calculated quantities is necessary.

\section{REFERENCES}

1. Boerner, W.-M., H. Mott, E. Lüneburg, B. Brisco, R. Brown, J. S. Paterson (authors); S. R. Cloude, E. Krogager, J. S. Lee, D. L. Schuler, J. J. van Zyl, D. Randall, and P. Budkewitsch (contributing authors), "Polarimetry in remote sensing: Basic and applied concepts," Chapter 5 in R. A. Reyerson, ed. The Manual of Remote Sensing, 3rd Edition, American Society of Photogrammetry and Remote Sensing, ASPRS Publishing, Bethesda, MD, 1998. 
2. Mott, H., "Antennas for radar and communications," A Polarimetric Approach, John Wiley and Sons, New York, 1992. \{Please, see his updated book Remote Sensing with Polarimetric Radar, Published with IEEE Press and Wiley-Interscience., to be released by November 2006\}.

3. Lüneburg, E., IEICE Transactions on Electronics (Special Issue on Electromagnetic Theory), E78-C 10, 1339, 1995.

4. Jolliffe, I. T., Principal Component Analysis, 2nd edn., Springer, New York, 2002.

5. Danklmayer, A., M. Chandra, and E. Lueneburg, "Principal component analysis in radar polarimetry," URSI, Kleinheubacher Berichte 2004, Advances in Radio Science, Vol. 3, 2005, in press.

6. Cloude, S. R. and E. Pottier, "A review of target decomposition theorems in radar polarimetry," IEEE Trans. GRS, Vol. 34, No. 2, 498-518, Mar. 1996.

7. Horn, R. A. and C. R. Johnson, Matrix Analysis, Cambridge University Press, New York, 1985.

8. Lüneburg, E. and W.-M. Boerner, "Statistical aspects of radar polarimetry," 43-52 in: P. Russer, M. Mongiardo (Editors), Fields, Networks, Computational Methods, and Systems in Modern Electrodynamics A Tribute to Leopold B. Felsen (the Proceedings of the Symposium on Fields, Networks and Computation: A Modern View of Engineering Electrodynamics, 2004 June 01-02, Technical University of Munich (in dedication to Leopold B. Felsen), SpringerVerlag Berlin-Heidelberg-New York, 2004.

9. Cloude, S. R., "Radar target decomposition theorems," Electronics Letters, Vol. 21, No. 1, 22-24, 1985.

10. Cloude, S. R., "Uniqueness of target decomposition theorems in radar polarimetry," in Boerner, W.-M. et al. (eds), Proceedings of the NATO Advances Research Workshop on Direct And Inverse Methods in Radar Polarimetry, Bad Windsheim, Germany, September 18-24, 1988; Kluwer Academic Publishers, Dordrecht 1992; NATO ASI Series C, Vol. 350, 267-296, 1988.

11. Cloude, S. R., "Polarimetric optimization based on the target covariance matrix," Electronics Letters, Vol. 26, No. 20, 1990.

12. Swartz, A. A., H. A. Yueh, J. A. Kong, L. M. Novak, and R. T. Shin, "Optimal polarizations for archiving maximum contrast in radar images," J. Geophys. Research, Solid Earth and Planets, Vol. 93, 15, 252-15, 260, 1988.

13. Cloude, S. R. and E. Pottier, "An entropy based classification scheme for land applications of polarimetric SAR," IEEE Trans. Geosci. Remote Sensing, Vol. 35, No. 1, 68-78, 1997. 